\title{
MRI-based skeletal hand movement model
}

\author{
Georg Stillfried, Ulrich Hillenbrand, Marcus Settles and Patrick van der Smagt
}

This paper is part of the book: Ravi Balasubramanian and Veronica Santos (editors), The Human Hand: A Source of Inspiration for Robotic Hands. Springer Tracts on Advanced Robotics, 2013. In print.

\begin{abstract}
The kinematics of the human hand is optimal with respect to force distribution during pinch as well as power grasp, reducing the tissue strain when exerting forces through opposing fingers and optimising contact faces. Quantifying this optimality is of key importance when constructing biomimetic robotic hands, but understanding the exact human finger motion is also an important asset in, e.g., tracking finger movement during manipulation. The goal of the method presented here is to determine the precise orientations and positions of the axes of rotation of the finger joints by using suitable magnetic resonance imaging (MRI) images of a hand in various postures. The bones are segmented from the images, and their poses are estimated with respect to a reference posture. The axis orientations and positions are fitted numerically to match the measured bone motions. Eight joint types with varying degrees of freedom are investigated for each joint, and the joint type is selected by setting a limit on the rotational and translational mean discrepancy. The method results in hand models with differing accuracy and complexity, of which three examples, ranging from 22 to $33 \mathrm{DoF}$, are presented. The ranges of motion of the joints show some consensus and some disagreement with data from literature. One of the models is published as an implementation for the free OpenSim simulation environment. The mean discrepancies from a hand model built from MRI data are compared against a hand model built from optical motion capture data.
\end{abstract}

Index Terms-human hand, robot hand, hand kinematics, MR imaging, 3D object localisation

G. Stillfried, U. Hillenbrand and P. v. d. Smagt are with the Institute of Robotics and Mechatronics, German Aerospace Center (DLR), Wessling, Germany. E-mail: \{georg.stillfried,ulrich.hillenbrand,smagt\}@dlr.de

M. Settles is with Klinikum rechts der Isar, university hospital of TU München, Munich, Germany. E-mail: msettles@tum.de

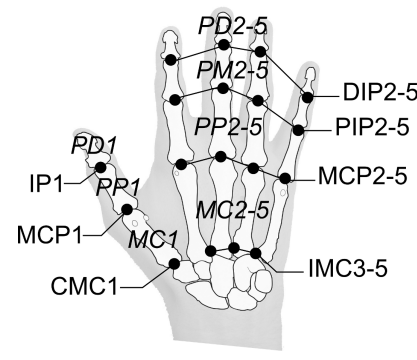

Fig. 1. The joints and bones of the fingers, the thumb and the metacarpus that are investigated here. Bone contours adapted from [1].

\section{FREQUENTLY USED ABBREVIATIONS}

$\begin{array}{ll}\text { Bones } & \\ \text { MC } & \text { metacarpal bone } \\ \text { PP } & \text { proximal phalanx } \\ \text { PM } & \text { medial phalanx } \\ \text { PD } & \text { distal phalanx } \\ \text { Joints } & \\ \text { CMC } & \text { carpometacarpal joint } \\ \text { IMC } & \text { intermetacarpal joint } \\ \text { MCP } & \text { metacarpophalangeal joint } \\ \text { PIP } & \text { proximal interphalangeal joint } \\ \text { DIP } & \text { distal interphalangeal joint } \\ \text { IP1 } & \text { thumb interphalangeal joint }\end{array}$

$\begin{array}{ll}\text { Other } & \\ \text { DoF } & \text { degree(s) of freedom } \\ \text { LOOCV } & \text { leave-one-out cross-validation } \\ \text { MRI } & \text { magnetic resonance imaging } \\ \text { MoCap } & \text { (optical) motion capture }\end{array}$

The abbreviations for bones and joints are augmented by numbers from 1 for thumb to 5 for little finger. For the location of the joints and bones, see Figure 1. Abbreviations for the joint types are found in Figure 6.

\section{INTRODUCTION}

Many robot hands have been built after the human example, one of the latest being the DLR Hand Arm System [2] (Figure 2). The design of its kinematics was guided by simple length measurements of a human hand, by functional considerations (e.g. how do the joint axes 


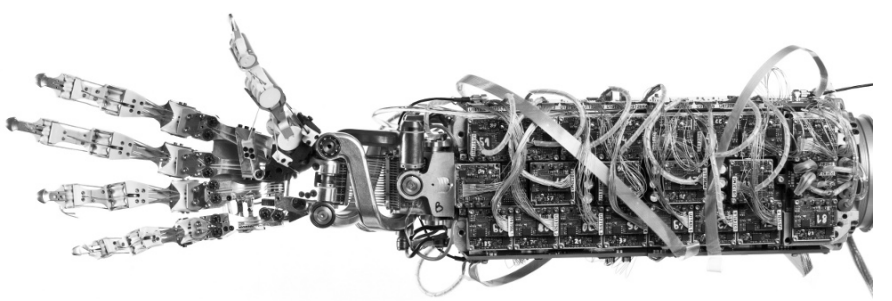

Fig. 2. Hand, wrist and forearm of the DLR Hand Arm System [2].

need to be inclined in order to achieve a robust opposition grasp) and by intuitive appeal of different models to human subjects [3]. Each movement degree of freedom (DoF) of the hand is supplemented by a stiffness DoF, so that 21 movement DoF of the hand and wrist are driven by 42 antagonistically placed motors. By tensioning nonlinear spring elements between the motors and the joints, the mechanical stiffness can be adjusted. This allows to mimic humans' stiffness variation in manipulation tasks, to store energy, e.g. for finger flicking, and to survive heavy collisions. With the motors placed in the robotic "forearm", the hand is embedded in a Hand Arm System, which provides five additional movement DoF for shoulder, elbow and forearm rotation.

Here we present a method that aims at very precisely measuring and modeling movement of the human hand skeleton, in order to verify and further improve robot hand kinematics. The method is demonstrated by creating hand models that cover all joints of fingers and thumb as well as the palm arching movement of the metacarpus (Figure 1). The models are based on magnetic resonance imaging (MRI) of an individual hand in 51 different postures, from which bone poses are extracted, localised, and used to optimise a parametrised general hand model.

Existing methods for modelling hand kinematics are mostly based on cadaver measurements [4]-[9] and optical surface tracking [10]-[17].

In our opinion, measurements at hand cadavers cannot be used to reconstruct the active kinematics of the hands, since the muscle synergies cannot be taken into account, plus the fixture by the ligaments is no longer realistic; consequently, such models will lead to artefacts in the kinematic representation. Models based on tracking the surface of the fingers, on the other hand, lead to unknown inaccuracies due to non-linear and varying movement of the soft tissue (e.g., the skin) with respect to the bones[18], [19]; indeed, such models often ignore phalanx rotation around their longitudinal axis. To overcome these disadvantages, we measure bone poses in vivo using $3 \mathrm{D}$ medical imaging. We conjecture that our method therefore leads to more accurate models of
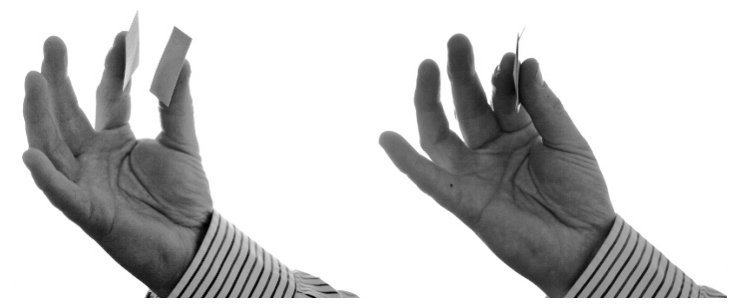

Fig. 3. Human hand kinematics enables optimal grasps by aptly orienting surfaces with respect to each other. Here the finger pads of thumb and index finger are brought face-to-face.

human hands.

The first and probably only previous work that used MRI images for measuring finger kinematics is by Miyata et al. [20]. They recorded one reference posture and three other postures to determine helical movement axes for the flexion axes of the proximal and distal interphalangeal joints of the index finger (PIP2 and DIP2).

We aim to extend the MRI-based kinematic analysis in three ways:

1) covering all joints of the hand, including multiDoF joints;

2) covering the range of motion of all the joints; and

3) constructing a continuous representation of the hand kinematics from which any intermediate posture can be generated.

For solving these issues, we need to reproducibly measure the movement of fixed parts of the fingers, i.e., the bones.

We model the human hand as a kinematic chain with an arbitrary number of DoF per joint. The optimal number of DoF for each joint, as well as the static parameters of each of these joints, are optimised from the recorded data. Once these parameters are determined for each of the joints, a model is generated with a fixed number of DoF. Since the model is targeted towards the subsequent implementation in a robotic system [21], only rotary joints are considered-any more complex joint in a robotic system will probably create friction and control difficulties.

Data recording is focussed on single subjects rather than statistical averages. When reproducing the kinematics of the human hand, statistics are of no great help: that approach would average over a number of participants without taking principal components of the variation into account. Instead, the kinematics of one adult individual is measured and reproduced.

The resulting model is made available within the OpenSim simulation environment [22]. OpenSim is a software that is able to match motion capture data to 
skeletal models, and can be extended to include tendons and muscles. Much of the human skeleton is already covered: legs, torso, neck, arms and a cadaver-based thumb and index finger [23]. The hand model presented here will be a step towards the completion of the human skeletal model.

\section{MethodS}

In order to determine the kinematics of the human hand, the rigid bones-forming the endoskeletal structure of the hand-are assumed as key reference points in the kinematics. The active kinematics is investigated, i.e., as induced by actively moving the joints through muscle activation, but passive kinematics, i.e., as induced by putting external forces on the fingers of the hand, is ignored. The presented reconstruction of the active kinematics of the human hand is based on the following scheme:

1) first, 3-dimensional MRI images of the hand are recorded in a large number of predefined postures (Section II-A). The postures must be chosen so as to represent the full kinematic range of the hand. Among a large number of other postures, the Kapandji Test [24] for the assessment of thumb motion is used;

2) second, in each 3-D MRI image the bones are segmented, using automatic grey-level-based segmentation followed by manual correction;

3) third, the position and orientation of each of the bones of the hand is determined. This is done automatically using novel image-processing algorithms, in which statistical methods are used to localise known objects in a 3-dimensional visual scene (Section II-B);

4) finally, from a range of possible joint models (Section II-C), the optimal model for each joint is selected and the parameters of the joints are determined in order to minimise the errors in the model (Section II-D). From that, kinematic chains are defined for each of the fingers, thus ending up with the full kinematic model.

The results of these steps are presented in Section III.

\section{A. MRI images and segmentation}

The MRI images are taken on a Philips Achieva $1.5 \mathrm{~T}$ unit with a Philips SENSE eight-channel head coil to receive a more homogeneous signal and to improve the signal-to-noise ratio (SNR). Generally, SNR is proportional to the voxel ${ }^{1}$ volume and to the square root of the

\footnotetext{
${ }^{1}$ voxel "volume pixel" = basic volume element of a 3-D image; analogous to pixel in 2-D images
}

net scan duration:

$$
r \propto v \sqrt{t}
$$

where $r$ is the SNR, $v$ is the voxel volume and $t$ is the net scan duration, i.e., the time actually spent for signal acquisition. Thus for every application an individual compromise has to be found optimally balancing the needs for a small $v$ (high spatial resolution), small $t$ (short scan times to minimise potential motion artefacts) and large $r$ (image quality sufficient for either diagnosis or-as in this case-the segmentation of certain anatomic structures).

An optimal compromise is found with a total scan duration (which is always longer than the net scan duration) of between two and two and a half minutes and a spatial resolution of $(0.76 \mathrm{~mm})^{3}$. Note that, from Equation (1), a voxel volume of $(0.38 \mathrm{~mm})^{3}$ would require 64 times the scan duration in order to achieve the same SNR. To further minimise motion artefacts the hand is stabilised using modelling clay. For postprocessing, the spatial resolution is interpolated to $(0.38$ $\mathrm{mm})^{3}$ in order to achieve sub-voxel resolution in the segmentation process. In the processing step after the segmentation, the grey value information is discarded. The interpolation helps retain some of the information that is contained in the grey values.

For scanning, the sequence type balancedFFE is used (also known as trueFISP or balancedSSFP) with $T_{\mathrm{R}} / T_{\mathrm{E}} /$ flip angle $=4.8 \mathrm{~ms} / 2.4 \mathrm{~ms} / 45^{\circ}$. The repetition time $T_{\mathrm{R}}$ is the time between two successive excitation pulses. The transverse component of the magnetization is read out at echo time $T_{\mathrm{E}}$ after each pulse.

The advantage of balancedFFE is that it yields a strong signal at short $T_{\mathrm{R}}$. (In fact, the signal of the balancedFFE sequence becomes independent of $T_{R}$, which can be as low as $2.5 \mathrm{~ms}$ with the limiting factors being the readout time and the avoidance of peripheral and heart muscle stimulation.)

As a drawback, balancedFFE is prone to the so-called banding artefacts appearing as black stripes across the bone. This artefact can in principle be overcome by applying the balancedFFE offset averaging technique (also known as CISS or FIESTA-C), but requires twice the scan time.

Another artefact occurring in these sequences is opposed phase fat/water cancelling, where voxels containing both fat and water, i.e. at corresponding tissue boundaries, appear dark, because the magnetisation vectors of fat and water point in opposite directions.

Also a cine-sequence, i.e. a continuous-motion sequence with two to five images per second, is recorded. However, only one image layer for the whole hand can 

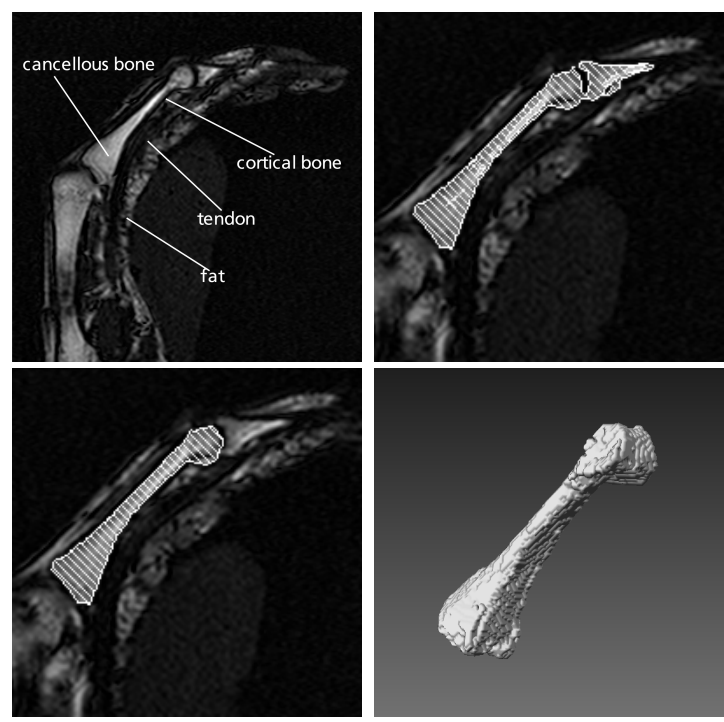

Fig. 4. Segmentation process. Top left: Slice of an MRI image, showing the middle finger metacarpal (MC3). Tissue types can be discriminated by the intensity of the signal that is emitted. Segmentation is done at the boundary between cancellous and cortical bone. Top right: Threshold-based preselection. Bottom left: Manually refined selection. Bottom right: Segmented volume consisting of the selected areas from all slices.

be recorded, which renders this method unusable for the purpose of exact bone localisation.

The images are taken of a 29 year old female subject with no history of hand problems who gave informed consent to the procedure. Fifty images are taken in different hand postures with the aim of reflecting each joint's range of motion.

From the MRI volume images, the bones are segmented. In fact, not the whole bone volume is segmented but the signal-intense volume inside the bone that corresponds to the cancellous bone. The tissue between the trabeculae of the cancellous bone is bone marrow consisting mainly of fat, which yields high signal intensity in the balancedFFE sequence.

The cortical bone, which forms the outer calcified layer of the bone, hardly contains any free fat or water protons and therefore stays dark in the MRI image. Near the bones there are other low-signal structures like tendons, which makes it difficult to determine the outer bone surface. Therefore, the boundary between cancellous and cortical bone is used for segmentation (Figure 4).

The bones are segmented from the image by highlighting the cancellous bone area in each slice of the MRI image. In the medical imaging software Amira (Visage Imaging $\mathrm{GmbH}$, Berlin, Germany), the area is preselected by adjusting a threshold and refined manually (Figure 4).

\section{B. Motion estimation}

For the purpose of estimating the rigid motion of bones between different hand postures, some geometric structure rigidly related to each bone has to be extracted from the MRI images that can be reliably recovered with little shape variation between images. Automatic reconstruction of the bone geometry is a challenge, as the image density of cancellous bone, cortical bone and surrounding tissue can vary greatly between and across images. Also manual segmentation, besides being tedious work, is prone to introducing shape variation.

Hence a double strategy is pursued. The border between cancellous and cortical bone often produces a marked contrast edge at reproducible locations. These border points can hence be detected by selection of highcontrast points. In the absence of such a marked density contrast, on the other hand, guidance by manual bone segmentation is needed. This double strategy is implemented as follows. First, the bone segments are padded with zero-density voxels to fit in a cuboid volume. Then a dipolarity score of the padded density within each $3 \times 3 \times 3$-voxel sub-volume is computed, as

$$
\begin{aligned}
& \operatorname{Dipolarity}\left(c_{1}, c_{2}, c_{3}\right)= \\
& \sum_{\substack{(i, j, k) \in\left\{c_{1}-1, c_{1}+1\right\} \\
\times\left\{c_{2}-1, c_{2}+1\right\} \\
\times\left\{c_{3}-1, c_{3}+1\right\}}}\left[I(i, j, k)-I\left(c_{1}, c_{2}, c_{3}\right)\right]\left(\begin{array}{l}
i \\
j \\
k
\end{array}\right) \| .
\end{aligned}
$$

Here $I(i, j, k)$ is the MRI image density as function of the voxel indices $(i, j, k)$, and $\left(c_{1}, c_{2}, c_{3}\right)$ are the indices of the centre voxel within the considered $3 \times 3 \times 3$ voxel sub-volume. The sum computes the difference vector of the density-weighted centroids of voxels with density higher and lower than the density $I\left(c_{1}, c_{2}, c_{3}\right)$ at the centre voxel; its Euclidean norm quantifies the degree of dipolarity of the density at the centre voxel. It attains high values for centre voxels close to a strong density edge. Finally, the centre voxels with the top $q$ percent of dipolarity are selected as representing bonerelated points. The grey value information is discarded in the selected points, but the interpolation mentioned in section II-A is used to refine the point set. The quantile $q$ is chosen to produce a data set of between 2,000 and 20,000 points, depending on the size of the bone. This way, points on the manually determined bone border are selected in the absence of high-contrast edges in the image; while high-contrast image edges dominate the selected points where available.

The above procedure produces sets of points that are close to the surface of the bones. However, missing 
parts and shape variation cannot be avoided. Moreover, there is no correspondence of points across different data sets of the same bone. A robust estimator of motion between such data sets hence has to be employed. A correspondence-free alignment that is also robust to geometric deviations [25] is provided within the framework of parameter-density estimation and maximization, or parameter clustering. This is a robust estimation technique based on location statistics in a parameter space where parameter samples are computed from data samples [26], [27]. The estimator may be viewed as a continuous version of a generalised, randomised Hough transform. In the present variant, samples are drawn from the 3D points selected through the high-dipolarity criterion above.

Let $X, Y \subset \mathbb{R}^{3}$ be the point sets extracted from two MRI images of the same bone. A motion hypothesis can be computed from a minimum subset of three points from $X$ matched against a minimum subset of three points from $Y$. The sampling proceeds thus as follows:

1) Randomly draw a point triple $\boldsymbol{x}_{1}, \boldsymbol{x}_{2}, \boldsymbol{x}_{3} \in X$;

2) Randomly draw a point triple $\boldsymbol{y}_{1}, \boldsymbol{y}_{2}, \boldsymbol{y}_{3} \in Y$ that is approximately congruent to the triple $\boldsymbol{x}_{1}, \boldsymbol{x}_{2}, \boldsymbol{x}_{3} \in$ $X$

3) Compute the rigid motion that aligns $\left(\boldsymbol{x}_{1}, \boldsymbol{x}_{2}, \boldsymbol{x}_{3}\right)$ with $\left(\boldsymbol{y}_{1}, \boldsymbol{y}_{2}, \boldsymbol{y}_{3}\right)$ in the least-squares sense;

4) Compute and store the six parameters of the hypothetical motion.

Random drawing of approximately congruent point triples in step 2 of the sampling procedure is efficiently implemented using a hash table of $Y$-point triples indexed with the three $X$-point distances $\left(\left\|\boldsymbol{x}_{1}-\boldsymbol{x}_{2}\right\|, \| \boldsymbol{x}_{2}-\right.$ $\left.\boldsymbol{x}_{3}\|,\| \boldsymbol{x}_{3}-\boldsymbol{x}_{1} \|\right)$ as the key. Least-squares estimation of rigid motion in step 3 computes the rotation $R \in \mathrm{SO}(3)$ and translation $\boldsymbol{t} \in \mathbb{R}^{3}$ as

$$
\begin{aligned}
(R, \boldsymbol{t})= & \underset{\left(R^{\prime}, \boldsymbol{t}^{\prime}\right) \in \mathrm{SE}(3)}{\arg \min }\left[\left\|R^{\prime} \boldsymbol{x}_{1}+\boldsymbol{t}^{\prime}-\boldsymbol{y}_{1}\right\|^{2}\right. \\
& \left.+\left\|R^{\prime} \boldsymbol{x}_{2}+\boldsymbol{t}^{\prime}-\boldsymbol{y}_{2}\right\|^{2}+\left\|R^{\prime} \boldsymbol{x}_{3}+\boldsymbol{t}^{\prime}-\boldsymbol{y}_{3}\right\|^{2}\right] .
\end{aligned}
$$

The special three-point method of [28] is used to obtain a closed-form solution. The parametrisation of rigid motions chosen for sampling step 4 may have an influence on the result. In fact, the parameter density from which the samples are taken depends upon this choice. A parametrisation that is consistent for clustering is used here, in the sense of [26].

By repeatedly executing the sampling procedure 1 4 above (in the order of $10^{6}$ times), samples are obtained from the parameter density for the rigid alignment problem. This parameter density is similar in spirit to a posterior density, but without assuming a probabilistic observation model.
The parameter samples can be stored in an array or a tree of bins. The sampling stops when a significant cluster of samples has formed, as judged from the bin counts. Then the location of maximum parameter density is searched by repeatedly starting a mean-shift procedure ([29], [30]) from the centre of the bins with high parameter counts. From all the local density maxima found through mean shift, the location in the $6 \mathrm{D}$ parameter space of the largest maximum is returned as the motion estimate of a bone, in the following denoted as $R_{\mathrm{e}}$ and $\boldsymbol{t}_{\mathrm{e}}$. Details of the implementation are presented elsewhere [27].

The main sources of error in the procedure for estimating bone motion are

- the variation in bone geometry erroneously represented in the point sets extracted from different images of the same bone, resulting from variation in manual segmentation or dipolarity values computed from the images;

- the approximate rotational symmetry about the longitudinal axis of a bone, especially in case of poor geometric representation lacking shape details.

To get rid of grossly wrong motion estimates, an interactive cluster analysis is performed on the estimated rotations. Making use of the stochastic nature of the estimation algorithm, each motion estimate is repeated 100 times with different subsets of the data being sampled, resulting in motion estimates $\left\{R_{\mathrm{e} 1}, \boldsymbol{t}_{\mathrm{e} 1}\right\} \ldots\left\{R_{\mathrm{e} 100}, \boldsymbol{t}_{\mathrm{e} 100}\right\}$. If the angular distance between any two of the 100 motion estimates exceeds a threshold, clusters of rotation parameters are identified and the correct cluster $\mathcal{C} \subset\{1, \ldots, 100\}$ is selected through visual inspection (Figure 5).

The angular distance between two rotations is defined as the angle of a third rotation that would have to be appended to the the first rotation in order to make it identical to the second rotation. It is calculated as follows:

$\operatorname{angdist}\left(R_{1}, R_{2}\right):=\arccos \left(\frac{1}{2}\left(\operatorname{trace}\left(R_{2} R_{1}^{-1}\right)-1\right)\right)$,

where $R_{1}$ and $R_{2}$ are the rotation matrices of the first and second rotation.

The final rotation estimate $\bar{R}$ is determined as the rotation that minimises the sum of squared angular distances to all rotations in the cluster, i.e., the mean rotation in the difference-rotation-angle metric,

$$
\bar{R}=\underset{R^{\prime} \in \operatorname{SO}(3)}{\arg \min }\left[\sum_{i \in \mathcal{C}} \operatorname{angdist}\left(R^{\prime}, R_{\mathrm{e} i}\right)^{2}\right],
$$

Likewise, the final translation estimate $\bar{t}$ is determined as the translation that minimises the sum of squared 


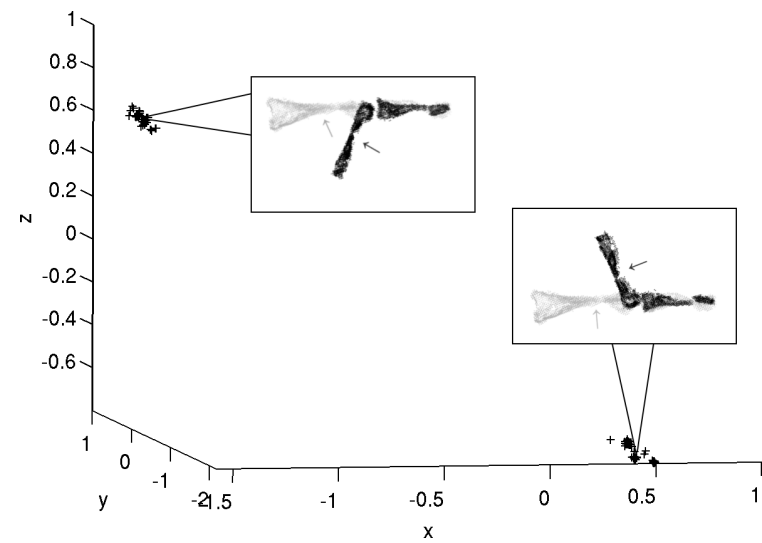

Fig. 5. Visual inspection of pose estimates. The rotational part of 100 randomly repeated pose estimates is plotted in three dimensions as the product of rotation axis and angle. In this example there are two distinct clusters. One element in each cluster is inspected by regarding the more strongly curved side of the neighbouring bone (arrows). The motion of the bottom right cluster element implies a large, anatomically impossible, longitudinal rotation of the bones. Therefore the top left cluster is taken as the correct cluster $\mathcal{C}$.

Euclidean distances to all translations in the cluster, i.e., the ordinary mean value of valid translations,

$$
\overline{\boldsymbol{t}}=\frac{1}{n} \sum_{i \in \mathcal{C}} \boldsymbol{t}_{\mathrm{ce} i}
$$

where $n$ is the number of elements in the correct cluster $\mathcal{C}$, and $\boldsymbol{t}_{\mathrm{ce} i}$ is the $i$-th translation estimate of the bone centroid. The translation estimate of the bone centroid is calculated as follows:

$$
\boldsymbol{t}_{\mathrm{ce} i}=R_{\mathrm{e} i} \boldsymbol{c}+\boldsymbol{t}_{\mathrm{e} i}-\boldsymbol{c},
$$

where $\boldsymbol{c}$ is the bone centroid, i.e. the mean of all points in $X$. If the correct cluster contains less than ten elements, the respective bone pose is discarded from the modelling process. Furthermore, all pose estimates are checked optically and obviously wrong estimates are discarded.

A natural confidence weight of the final rotation estimates is obtained from the variance of the sample mean values, i.e.,

$$
\sigma_{\mathrm{r}}^{2}=\frac{1}{n(n-1)} \sum_{i=1}^{n} \operatorname{angdist}\left(\bar{R}, R_{\mathrm{e} i}\right)^{2} .
$$

This confidence weight enters in the estimation of orientation of rotational axes for the kinematic hand model below. Likewise, a confidence weight of the final translation estimates is given by

$$
\sigma_{\mathrm{t}}^{2}=\frac{1}{n(n-1)} \sum_{i=1}^{n}\left\|\overline{\boldsymbol{t}}-\boldsymbol{t}_{\mathrm{ce} i}\right\|^{2},
$$

and used in the estimation of the position of rotational axes for the kinematic hand model below.

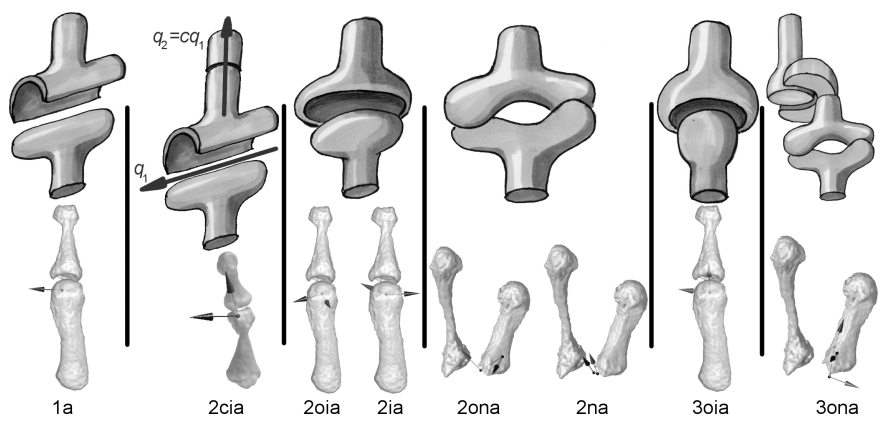

Fig. 6. Joint types used in the presented method. From left to right: Hinge joint (one axis, "1a"), hinge joint with combined longitudinal rotation (two coupled intersecting axes, "2cia"), condyloid joint (two orthogonal/oblique intersecting axes, "2oia"/"2ia"), saddle joint (two orthogonal/oblique non-intersecting axes, "2ona"/“2na"), ball joint (three orthogonal intersecting axes, "3oia") and 3-DoF joint with orthogonal non-intersecting axes ("3ona", combination of a saddle and a pivot joint). Upper row images from [31].

\section{Determining joint models}

In the fingers of the human hand contain different types of joints. The 1-DoF joints all are hinge joints (Figure 6); 2-DoF joints can be divided into two types. The metacarpal joint of the thumb is a saddle joint. In contrast, the metacarpal joints of the fingers are condyloid. The main difference between saddle and condyloid joints is that condyloid joints have (roughly) intersecting axes, which saddle joints do not have.

The condyloid and saddle joint types are further divided into joints whose rotation axes are orthogonal and joints whose rotation axes are at any arbitrary angle to each other. Additionally, a hinge joint with a coupled longitudinal rotation, a ball joint and a 3-DoF joint with non-intersecting axes are defined (Figure 6).

As mentioned above, one of the goals is to compute an optimal kinematic model for a robotic system. For that reason, but also for reasons of computational efficiency and easy representation, the joints are rotational joints with axes fixed to the proximal bone or the preceding axis in multi-DoF joints. This certainly has an effect on the accuracy of the model, but this accuracy remains within the accuracy of the recording and reconstruction method.

Typically there is a trade-off between the complexity and the accuracy of a joint type. For each joint, the joint type is selected by setting a limit on the mean deviation between the measured and modelled bone poses, and by selecting the simplest joint type that fulfills it. Joints that have fewer axes are considered simpler than joints with more axes, intersecting axes simpler than nonintersecting axes and orthogonal axes simpler than freely oriented ones. The mean deviation is an outcome of the identification of the joint parameters (Section II-D). 


\section{Identification of joint parameters}

The joint parameters (positions and orientations of the rotation axes) are identified on a joint-by-joint basis by numerically minimising the discrepancy between the measured and modelled relative motion of the joint's distal bone with respect to the proximal bone. To calculate the relative motion, the absolute motion of the proximal bone is inversely applied to the absolute motion of the distal bone:

$$
R_{\mathrm{r}}=\bar{R}_{\mathrm{p}}^{-1} \bar{R}_{\mathrm{d}}
$$

and

$$
\boldsymbol{t}_{\mathrm{r}}=\bar{R}_{\mathrm{p}}^{-1}\left(\boldsymbol{c}_{\mathrm{d}}+\overline{\boldsymbol{t}}_{\mathrm{d}}-\overline{\boldsymbol{t}}_{\mathrm{p}}-\boldsymbol{c}_{\mathrm{p}}\right)+\boldsymbol{c}_{\mathrm{p}}-\boldsymbol{c}_{\mathrm{d}},
$$

where $\left\{R_{\mathrm{r}}, \boldsymbol{t}_{\mathrm{r}}\right\}$ is the relative motion of the distal bone with respect to the proximal bone, $\left\{\bar{R}_{\mathrm{p}}, \overline{\boldsymbol{t}}_{\mathrm{p}}\right\}$ and $\left\{\bar{R}_{\mathrm{d}}, \overline{\boldsymbol{t}}_{\mathrm{d}}\right\}$ are the absolute motions of the proximal and distal bone according to Equations (3) and (4), and $\boldsymbol{c}_{\mathrm{p}}$ and $\boldsymbol{c}_{\mathrm{d}}$ are the vectors of Cartesian coordinates of the centroids of the proximal and distal bone.

In order to reduce the dimensionality of the search space, the identification of the axis orientations and positions is split up into two steps. In the first step, the axis orientations are identified by minimising the angular difference between the measured orientations and the modelled orientations.

The modelled orientation $R_{\mathrm{m}}$ of the bone is calculated as follows:

$$
R_{\mathrm{m}}=\prod_{k=1}^{n_{\mathrm{a}}} \operatorname{rot}\left(\boldsymbol{a}_{k}, q_{k}\right)
$$

where $n_{\mathrm{a}} \in\{1,2,3\}$ is the number of rotation axes of the joint, $\boldsymbol{a}_{k}$ is the orientation of the $k^{\text {th }}$ axis and $q_{k}$ is the rotation angle around the $k^{\text {th }}$ axis. The operator $\operatorname{rot}(\boldsymbol{a}, q)$ yields the rotation matrix of a rotation around an axis $\boldsymbol{a}$ by an angle $q$ :

$$
\begin{aligned}
& \operatorname{rot}(\boldsymbol{a}, q):= \\
& \left(\begin{array}{ccc}
c+c^{\prime} a_{x}^{2} & c^{\prime} a_{x} a_{y}-a_{z} s & c^{\prime} a_{x} a_{z}+a_{y} s \\
c^{\prime} a_{x} a_{y}+a_{z} s & c+c^{\prime} a_{y}^{2} & c^{\prime} a_{y} a_{z}-a_{x} s \\
c^{\prime} a_{x} a_{z}-a_{y} s & c^{\prime} a_{y} a_{z}+a_{x} s & c+c^{\prime} a_{z}^{2}
\end{array}\right),
\end{aligned}
$$

with

$$
\begin{gathered}
c=\cos q, \\
c^{\prime}=1-\cos q \text { and } \\
s=\sin q,
\end{gathered}
$$

where $a_{x}, a_{y}$ and $a_{z}$ are the Cartesian elements of the unit orientation vector $\boldsymbol{a}$. The position and orientation vectors of the rotation axes are given in the coordinate system of the MRI system, and with respect to the bones in the reference posture.
The orientations of the rotation axes and the rotation angles are identified by numerically minimising the weighted mean square angular difference over all postures:

$$
\begin{gathered}
\left(\boldsymbol{a}_{1}, \ldots, \boldsymbol{a}_{n_{\mathrm{a}}}, \boldsymbol{q}_{1}, \ldots, \boldsymbol{q}_{n_{\mathrm{p}}}\right)=\underset{\left(\boldsymbol{a}_{1}^{\prime}, \ldots, \boldsymbol{a}_{n_{\mathrm{a}}}^{\prime}, \boldsymbol{q}_{1}^{\prime}, \ldots, \boldsymbol{q}_{n_{\mathrm{p}}}^{\prime}\right)}{\arg \min } \\
{\left[\sum_{j=1}^{n_{\mathrm{p}}} w_{\mathrm{r} j} \operatorname{angdist}\left(R_{\mathrm{r} j}, R_{\mathrm{m} j}\left(\boldsymbol{a}_{1}^{\prime}, \ldots, \boldsymbol{a}_{n_{\mathrm{a}}}^{\prime}, \boldsymbol{q}_{j}^{\prime}\right)\right)^{2}\right],}
\end{gathered}
$$

with

$$
w_{\mathrm{r} j}=\frac{1}{\sigma_{\mathrm{rp} j}^{2}+\sigma_{\mathrm{rd} j}^{2}}
$$

where $n_{\mathrm{p}}$ is the number of postures, $\boldsymbol{a}_{1}, \ldots, \boldsymbol{a}_{n_{\mathrm{a}}}$ are the orientation vectors of the rotation axes, $\boldsymbol{q}_{1}, \ldots, \boldsymbol{q}_{n_{\mathrm{p}}}$ are the vectors of joint angles for each posture $j \in$ $\left\{1, \ldots, n_{\mathrm{p}}\right\}$, where $\boldsymbol{q}_{j}=\left(q_{1 j}, \ldots, q_{n_{\mathrm{a}} j}\right)^{\mathrm{T}}$ contains the joint angles for each rotation axis, $w_{\mathrm{r} j}$ is the confidence weight due to the variances $\sigma_{\mathrm{rp} j}^{2}$ and $\sigma_{\mathrm{rd} j}^{2}$ of the rotation estimates of the proximal and distal bone in posture $j$ as calculated in Equation (5), angdist is the angular distance operator according to Equation (2), $R_{\mathrm{r} j}$ is the measured relative orientation of the bone in posture $j$ according to Equation (7) and $R_{\mathrm{m} j}$ is the modelled relative orientation of the bone according to Equation (9).

The positions of the rotation axes are identified by minimising the mean squared distance between the measured and modelled position of the bone centroid:

$$
\begin{aligned}
& \left(\boldsymbol{p}_{1}, \ldots, \boldsymbol{p}_{n_{\mathrm{a}}}\right)= \\
& \underset{\left(\boldsymbol{p}_{1}^{\prime}, \ldots, \boldsymbol{p}_{n_{\mathrm{a}}}^{\prime}\right)}{\arg \min }\left[\sum_{j=1}^{n_{\mathrm{p}}} w_{\mathrm{t} j}\left\|\boldsymbol{t}_{\mathrm{m} j}\left(\boldsymbol{p}_{1}^{\prime}, \ldots, \boldsymbol{p}_{n_{\mathrm{a}}}^{\prime}\right)-\boldsymbol{t}_{\mathrm{r} j}\right\|^{2}\right],
\end{aligned}
$$

with

$$
\boldsymbol{t}_{\mathrm{m} j}\left(\boldsymbol{p}_{1}^{\prime}\right)=\left(\prod_{k=1}^{n_{\mathrm{a}}} \operatorname{rot}\left(\boldsymbol{a}_{k}, q_{k j}\right)\right)\left(\boldsymbol{c}_{\mathrm{d}}-\boldsymbol{p}_{1}^{\prime}\right)+\boldsymbol{p}_{1}^{\prime}-\boldsymbol{c}_{\mathrm{d}}
$$

for joints with intersecting axes,

$$
\begin{aligned}
& \boldsymbol{t}_{\mathrm{m} j}\left(\boldsymbol{p}_{1}^{\prime}, \ldots, \boldsymbol{p}_{n_{\mathrm{a}}}^{\prime}\right)=\left(\prod_{k=1}^{n_{\mathrm{a}}} \operatorname{rot}\left(\boldsymbol{a}_{k}, q_{k j}\right)\right) \boldsymbol{c}_{\mathrm{d}}+ \\
& \left(\sum_{k=1}^{n_{\mathrm{a}}}\left(\prod_{l=1}^{k-1} \operatorname{rot}\left(\boldsymbol{a}_{l}, q_{l j}\right)-\prod_{l=1}^{k} \operatorname{rot}\left(\boldsymbol{a}_{l}, q_{l j}\right)\right) \boldsymbol{p}_{k}^{\prime}\right)-\boldsymbol{c}_{\mathrm{d}}
\end{aligned}
$$

for joints with non-intersecting axes and

$$
w_{\mathrm{t} j}=\frac{1}{\sigma_{\mathrm{tp} j}^{2}+\sigma_{\mathrm{td} j}^{2}},
$$


where $\boldsymbol{p}_{1}, \ldots, \boldsymbol{p}_{n_{\mathrm{a}}}$ are the position vectors of the rotation axes, $\boldsymbol{t}_{\mathrm{m} j}$ are the modelled translations of the bone centroid, $t_{\mathrm{r} j}$ are the measured translations of the bone centroid, $\boldsymbol{a}_{k}$ and $q_{k j}$ are the rotation axes and angles as derived from Equation (11) and $\boldsymbol{c}_{\mathrm{d}}$ is the position vector of the distal bone centroid.

In order to perform the optimisations described in Equations (11) and (13), the fminsearch function of the Matlab computation software is used, which implements the Nelder-Mead simplex algorithm [32]. The algorithm is called with broadly different starting points to increase the chance of finding the global optimum, and not only a local optimum. For Equation (11), a nested optimisation is conducted, with an outer optimisation for the axis orientations $\boldsymbol{a}_{1}, \ldots, \boldsymbol{a}_{n_{\mathrm{a}}}$. Within each iteration step of the outer optimisation, a number of $n_{\mathrm{p}}$ inner optimisations are carried out to find the optimum joint angles $\boldsymbol{q}_{1}, \ldots, \boldsymbol{q}_{n_{\mathrm{p}}}$. For the outer optimisation, the axis orientations are parametrised by two spherical coordinates (azimuth and elevation), in order to reduce the search space by one dimension as compared to Cartesian coordinates for axis orientation.

\section{E. Leave-one-out cross-validation of joint parameters}

In order to check to what extent the results apply to the investigated hand in general as opposed to being overfit to the investigated postures, a leave-one-out crossvalidation is performed. For this, the parameters of the joints are identified $n_{\mathrm{p}}$ times, with $n_{\mathrm{p}}$ being the number of measured bone poses, where in each round one of the poses is left out. The joint parameters (axis orientations and positions) resulting from each identification are used to move the bone as close as possible to the omitted pose. The rotational and translational discrepancy between the modelled and measured bone pose is calculated, and the weighted mean of rotational and translational discrepancies between the modelled and measured bone poses is calculated.

\section{F. Comparison with optical motion capture}

Kinematic hand models are built based on MRI data and based on optical motion capture (MoCap) data. The residual rotational and translational discrepancies of both models are compared.

Another subject is recruited for MRI and MoCap measurements, because the previous subject was not available anymore. Due to time constraints, only one reference posture and 19 other postures are recorded with MRI, using a turboFFE sequence and a spatial resolution of $(1 \mathrm{~mm})^{3}$. For MoCap, a Vicon system (OMG plc, Oxford, UK) with seven 0.3-megapixels cameras is used.
One finger is recorded at a time, with three retroreflective markers per finger segment. One reference time sample and nineteen representative other samples are selected from the capture data. In the reference frame, bone coordinate systems are attached manually to the marker triples of each segment. The motion of the finger segment from the reference posture to the other postures is determined using the least-sqares method by [28].

One joint instead of three joints is used to model the palm, because the motion of the single metacarpal bones is difficult to discriminate with MoCap. The same fifteen joints for fingers and thumb as described above are used. The thumb CMC joint is modelled with two nonorthogonal, non-intersecting axes (2na), the MCP joints are modelled with two orthogonal, intersecting axes (2oia) and the remaining joints are modelled with single axes (1a). The axis parameters and residual rotational and translational discrepancies are modelled as described above.

Additionally, whole finger postures are matched with both methods. For this, the joints are concatenated to form kinematic chains. The global pose and the joint angles are optimised to minimise the mean rotational and translational discrepancies between the modelled and measured bone poses. For this, a weighting between the rotational and translational discrepancy is decided. One millimetre of translational discrepancy is treated with the same weight as one degree of rotational discrepancy.

The means of the residual discrepancies are tested with a two-tailed Student's t-test for unpaired data, with a significance threshold $\alpha=5 \%$, to find out if they are statistically significantly different. We conjecture that the MRI method will lead to lower residuals than MoCap, because the measurements are not disturbed by soft tissue artefacts. The null hypothesis is that the mean residuals are equal.

\section{RESULTS}

The calculation steps described in the previous Methods section lead to optimised joint parameters. By setting a limit on the modelling error, the joint types for each joint are found (Section III-A). The modelling error is computed for each joint and checked by a leave-one-out cross-validation (Section III-B). The measurement error is assessed by a repeatability test (Section III-C). As far as available, results are compared to data from literature (Section III-D). The software implementation of the hand model is introduced (Section III-E).

\section{A. Joint types}

The main results of the presented method are movement models of the analysed human hand. Depending 
on the desired accuracy in terms of discrepancy between modelled and measured bone poses, hand models with different complexity are generated. In Figure 7, different hand models from simple (top) to complex (bottom) are presented.

In the simple model, four joints are modelled as 2-DoF universal joints: thumb, index, ring and little finger MCP. The other joints are modelled as 1-DoF hinge joints.

The intermediately complex hand model (middle) differs from the simple one by providing two DoF each to MCP3 and CMC1. The joint axes of MCP3 intersect, while the ones of $\mathrm{CMC} 1$ do not.

The most complex model (bottom) models CMC1 with three non-intersecting axes, with the third one allowing a longitudinal rotation (pro-/supination) of MC1. A longitudinal rotation is also enabled in DIP2 and PIP5, while PIP2 allows a combined longitudinal rotation and sidewards movement. The little finger DIP joint allows a longitudinal rotation only in an extended position. Additional DoF for sidewards movement are found in DIP2, DIP3, DIP4 and IP1.

The weighted-mean rotational deviation per joint ranges from $1.6^{\circ}$ in IMC3 to $5.5^{\circ}$ in IP1. The maximum rotational deviation in a single hand posture is $17.2^{\circ}$ in $\mathrm{CMC} 1$. Weighted-mean translational deviation ranges from $0.9 \mathrm{~mm}$ (PIP4) to $2.6 \mathrm{~mm}$ (CMC1), and the maximum translational deviation in a single hand posture is $7.2 \mathrm{~mm}$, and also occurs in CMC1. The examples in Figure 8 are supposed to give a feel of these values.

\section{B. Cross-validation of the results}

For most joints, there is only a slight increase of the rotational and translational modelling error from the whole data mean error (columns 3 and 5) to the LOOCV mean error. For example, in the thumb MCP joint, the mean angular when using all poses is $2.5^{\circ}$, and the mean angular error of the LOOCV is $2.9^{\circ}$. In the same joint, the mean translational error is $1.2 \mathrm{~mm}$ when taking into account all poses and $1.3 \mathrm{~mm}$ in the LOOCV analysis. This means that the results are generally valid for the investigated individual hand and do not depend on certain postures.

All differences for the translational error are within $0.2 \mathrm{~mm}$ and all differences for the rotational error are within $1.0^{\circ}$ except for the thumb interphalangeal joint, where the difference is $1.2^{\circ}$ and the little finger metacarpophalangeal joint, where it is $3.0^{\circ}$. In these exceptional cases the joint parameters depend strongly on the selection of the subset of bone poses. This means that there are single extreme poses is the data that are not adequately represented by the other poses.
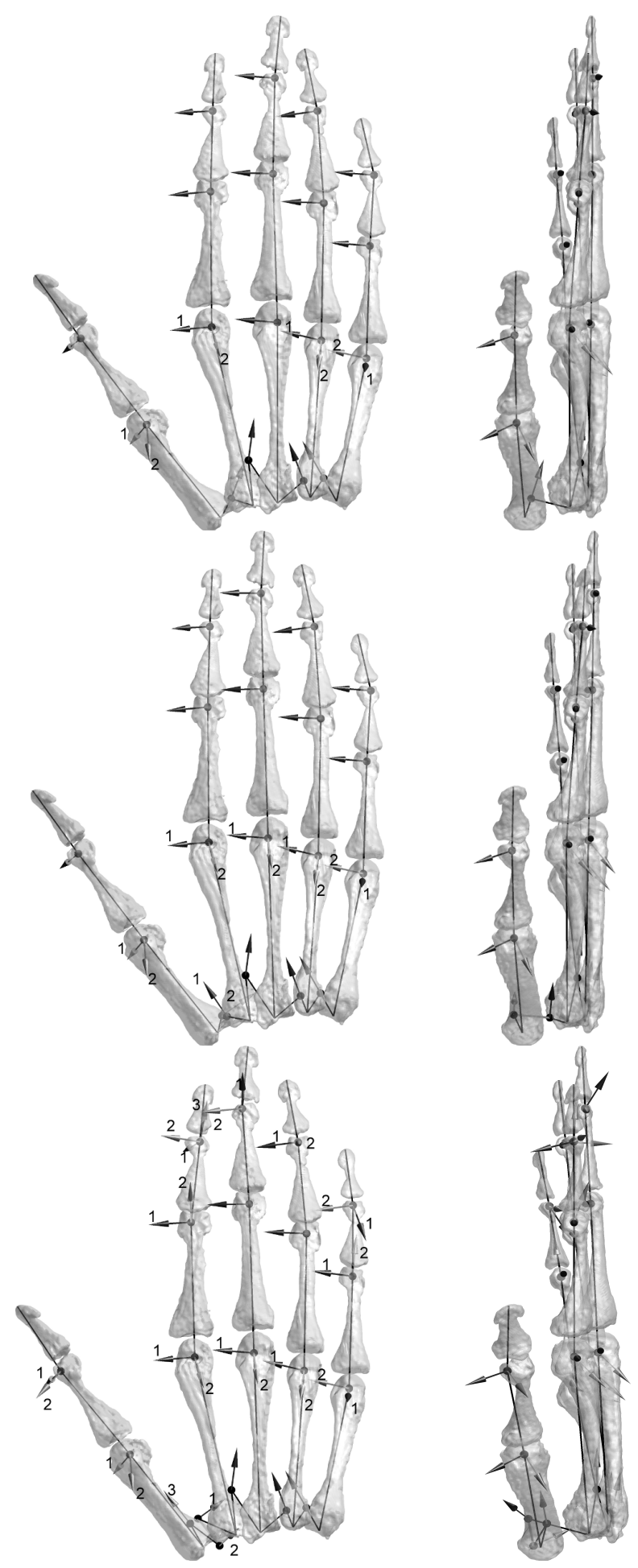

Fig. 7. Variants of the kinematic model at different accuracy constraints, dorsal view (left) and radial view (right).

Top: 22 DoF, rotational deviation $<9^{\circ}$, translational deviation $<$ $6 \mathrm{~mm}$. Middle: $24 \mathrm{DoF}$, rotational deviation $<6^{\circ}$, translational deviation $<3 \mathrm{~mm}$. Bottom: $33 \mathrm{DoF}$, rotational deviation $<3^{\circ}$, translational deviation $<2 \mathrm{~mm}$. In joints with more than one axis, the first one is marked " 1 ", the second one " 2 ", and, if existing, the third one " 3 ". 


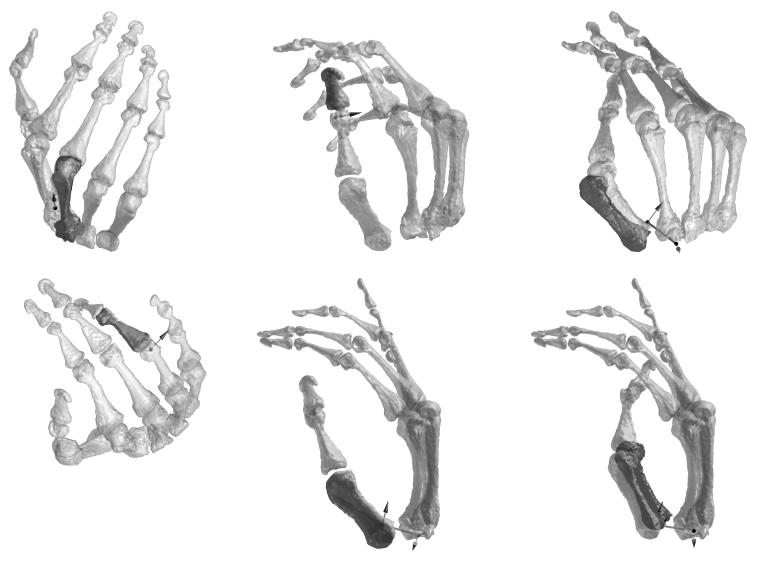

Fig. 8. Comparison of measured (bright) and modelled (dark) bone poses in several postures. Top left: Pose of the bone MC4 relative to MC3 in posture 36. The rotational discrepancy is $1.6^{\circ}$ and the translational discrepancy is $1.0 \mathrm{~mm}$. The arrow is the rotation axis of the modelled IMC4 joint that connects MC3 and MC4. Top middle: DP1 relative to PP1 in posture 1 . Discrepancy: $5.5^{\circ}, 1.4 \mathrm{~mm}$. IP1 joint. Top right: MC1 relative to MC2 in posture 29. Discrepancy: $17.2^{\circ}$, $6.4 \mathrm{~mm}$. CMC1 joint. Bottom left: MP4 relative to PP4. Discrepancy: $2.6^{\circ}, 0.9 \mathrm{~mm}$. PIP4 joint. Bottom middle: MC1 relative to MC2 in posture 24 . Discrepancy: $5.5^{\circ}, 2.6 \mathrm{~mm}$. Bottom right: $\mathrm{MC} 1$ relative to MC2 in posture 35. Discrepancy: $5.1^{\circ}, 7.2 \mathrm{~mm}$.

\section{Motion estimation repeatability}

The repeatability of the motion estimations is examined by repeating it 100 times with randomly permuted point sets. The standard deviation of the rotation and translation estimate is given in Table I as the square root of the variance described in Equations (5) and (6).

\section{Comparison with results from literature}

Comparing the range of motion (RoM) of the MCP joints with the values provided by [33], in most points the results agree, but some are different. [33] states that

1) the flexion RoM "falls short of $90^{\circ}$ for the index but increases progressively with the other fingers",

2) "active extension [...] can reach up to $30^{\circ}$ or $40^{\circ}$ " and

3) "the index finger has the greatest side-to-side movement".

In the hand we used in our measurements, a smaller flexion RoM for the index finger $\left(80^{\circ}\right)$ is measured, while there is an increase towards in the little finger: middle and ring finger are similar with $86^{\circ}$ and $84^{\circ}$, respectively, and the little finger has a flexion RoM of $95^{\circ}$. The active extension $\mathrm{RoM}$ is $30^{\circ}$ (index), $33^{\circ}$ (middle), $43^{\circ}$ (ring) and $52^{\circ}$ (little finger) and therefore higher than the ones in [33] for the ring and little finger. We agree that, for the hand we investigated, index finger side-toside movement is greater than that of the other fingers

\begin{tabular}{|c|c|c|c|c|c|c|}
\hline \multirow[b]{2}{*}{ bone } & \multicolumn{3}{|c|}{$\sigma_{\mathrm{r}}\left({ }^{\circ}\right)$} & \multicolumn{3}{|c|}{$\sigma_{\mathrm{t}}(\mathrm{mm})$} \\
\hline & $\min$ & $\max$ & mean & $\min$ & $\max$ & mean \\
\hline MC1 & 1.0 & 5.3 & 3.0 & 0.1 & 0.2 & 0.1 \\
\hline PP1 & 1.6 & 5.7 & 3.2 & 0.1 & 0.3 & 0.1 \\
\hline PD1 & 1.2 & 5.4 & 1.8 & 0.1 & 0.3 & 0.1 \\
\hline $\mathrm{MC} 2$ & 1.7 & 8.0 & 3.2 & 0.1 & 0.4 & 0.2 \\
\hline PP2 & 1.0 & 5.9 & 2.8 & 0.1 & 0.2 & 0.1 \\
\hline PM2 & 1.2 & 3.7 & 2.1 & 0.0 & 0.1 & 0.1 \\
\hline PD2 & 3.2 & 4.9 & 3.9 & 0.0 & 0.1 & 0.1 \\
\hline MC3 & 1.1 & 23.7 & 2.6 & 0.1 & 0.5 & 0.2 \\
\hline PP3 & 1.3 & 5.8 & 3.1 & 0.1 & 0.2 & 0.1 \\
\hline PM3 & 1.0 & 2.9 & 1.7 & 0.0 & 0.1 & 0.1 \\
\hline PD3 & 2.9 & 5.1 & 3.3 & 0.1 & 0.4 & 0.2 \\
\hline MC4 & 1.4 & 7.6 & 3.3 & 0.1 & 0.5 & 0.1 \\
\hline PP4 & 0.9 & 8.9 & 3.4 & 0.1 & 0.2 & 0.1 \\
\hline PM4 & 1.2 & 8.5 & 2.5 & 0.0 & 0.4 & 0.1 \\
\hline PD4 & 2.1 & 4.3 & 2.9 & 0.1 & 0.2 & 0.1 \\
\hline MC5 & 1.4 & 11.2 & 3.6 & 0.1 & 0.4 & 0.1 \\
\hline PP5 & 1.5 & 7.1 & 3.3 & 0.0 & 0.1 & 0.1 \\
\hline PM5 & 1.0 & 4.3 & 2.8 & 0.0 & 0.1 & 0.1 \\
\hline PD5 & 0.1 & 4.4 & 3.1 & 0.0 & 0.1 & 0.0 \\
\hline all & 0.1 & 23.7 & 2.9 & 0.0 & 0.5 & 0.1 \\
\hline
\end{tabular}

STANDARD DEVIATION OF THE MOTION ESTIMATION FOR THE ROTATIONAL $\left(\sigma_{\mathrm{r}}\right)$ AND TRANSLATIONAL $\left(\sigma_{\mathrm{t}}\right)$ PART. THE MINIMUM, MAXIMUM AND MEAN OVER ALL IMAGES ARE GIVEN.

with $59^{\circ}$ (index), $43^{\circ}$ (middle), $44^{\circ}$ (ring) and $54^{\circ}$ (little finger).

For the PIP and DIP joint, Kapandji states that

1) the "range of flexion in the PIP joints is greater than $90^{\circ}$ " and

2) in the DIP joints "slightly less than $90^{\circ}$ ",

3) the range "increases from the second to fifth finger" to $135^{\circ}$ (PIP5) and

4) to "a maximum of $90^{\circ}$ " (DIP5);

5) the range for PIP extension is zero and

6) for active DIP extension zero or trivial.

Our measurements agree with points 1 and 2. An increase from second to fifth finger (points 3 and 4) is not observed. Small PIP and DIP extension angles (up to $22.5^{\circ}$ ) are measured.

\section{E. OpenSim}

The OpenSim model of the hand contains simplified bone geometries and the full set of joints. The joints can be moved by sliders (Figure 9). The OpenSim framework also provides for the extension of the model with tendons and muscles [22]. The model is available for download as a zip-compressed file at http://www.robotic.dlr.de/ human_hand. 


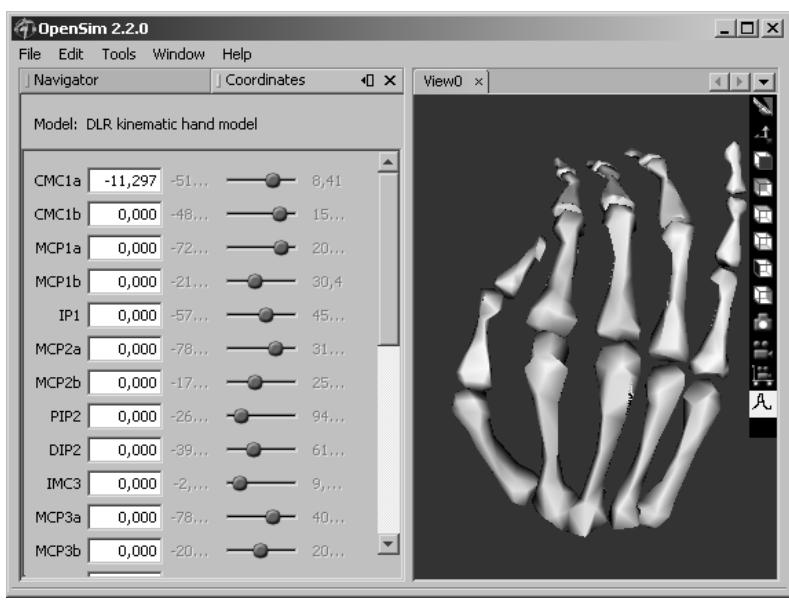

Fig. 9. Screenshot of the OpenSim application. Each DoF of the skeletal model can be moved by a slider.

\section{F. Dependence of results on starting points}

The result of the parameter identification is in some joints sensitive to the optimisation starting point and in others not. For example, the parameters of the CMC1 joint were optimised with three different starting points for each of the two axis orientations and three different starting points for the axis offset. The results are slightly sensitive to the axis orientation starting points, with rotational error ranging from $2.95^{\circ}$ to $3.18^{\circ}$. The variation of the axis offset starting point has no effect on the results. In other joints, for example the IP1 joint, the optimisation starting points have no effect on the result.

\section{G. MRI versus MoCap}

MRI and optical motion capture (MoCap) were compared with respect to the residual discrepancies, as described in Section II-F. The results of this comparison are shown in Figure 10. The mean rotational residual for single joints is $4.4^{\circ}$ for MRI data explained by the MRI-based model and $4.7^{\circ}$ for MoCap data explained by the MoCap-based model. The mean translational residual for single joints is $1.4 \mathrm{~mm}$ (MRI) and $1.2 \mathrm{~mm}$ (MoCap) respectively. The mean rotational residual for whole fingers is $3.5^{\circ}$ and $3.6^{\circ}$, respectively. The mean translational residual for whole fingers is $1.2 \mathrm{~mm}$ and $1.5 \mathrm{~mm}$, respectively.

The null hypothesis that the data come from distributions with the same mean could not be rejected except in the case of the translational residual of single joints. The mean translational residual is statistically significantly smaller for MoCap than for MRI.

\section{DISCUSSION}

We showed that MRI images can be used to build a movement model of the human hand. Estimating the

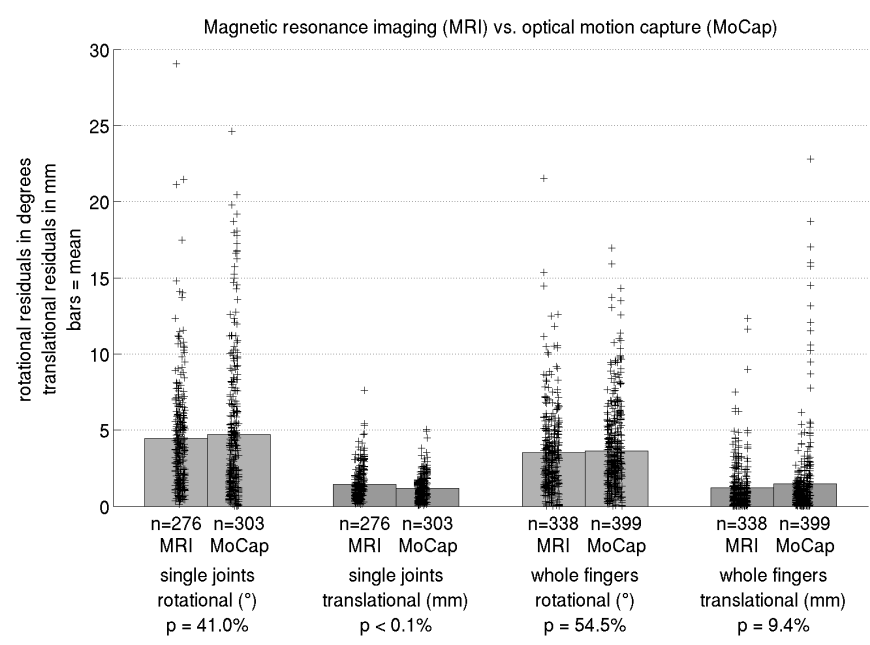

Fig. 10. Comparison of MRI and MoCap. On the left, single bone poses are measured, and on the right, whole finger postures. The points marked by "plus" signs show the residual rotational discrepancies in degrees and the translational discrepancies in $\mathrm{mm}$, respectively, of each bone pose in each posture. The bars show the mean values. The value $n$ is the number of bone poses, and the $p$-value is the probability that a difference of means equal or larger than the observed one would occur if data were sampled from normal distributions with an identical mean.

longitudinal orientation of the bones proved difficult, but reasonable accuracy could be achieved by repeated measurements. The resulting model can be used for visualising skeletal motion based on motion capture data, using the freely available software OpenSim.

It should be borne in mind that the presented models are based on one individual hand. Since different people have different hands in terms of e.g. size, flexibility and possibly kinematics, the models should be used with caution. In OpenSim there is, however, a functionality to scale skeletal models according to measured marker movements. This might compensate part of the interindividual differences.

\section{A. Consideration of errors}

There are six sources for errors in the kinematic modelling process:

1) selection of postures,

2) MRI acquisition,

3) segmentation,

4) motion estimation,

5) joint definition, and

6) joint parameter identification.

It is impossible to consider all possible postures of each joint as they are infinite. Ideal, therefore, would be a very dense sampling of postures during a large number of different movements. This is not possible in MRI due to cost and time constraints. Hand postures for this 


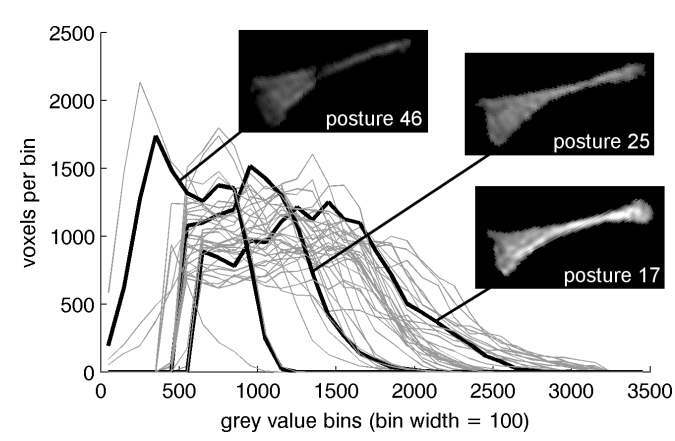

Fig. 11. Histograms of the grey value distributions of middle finger medial phalanx in different segmented MRI images. Three clearly different examples are highlighted. Of these the central sagittal slice of the MRI image is shown.

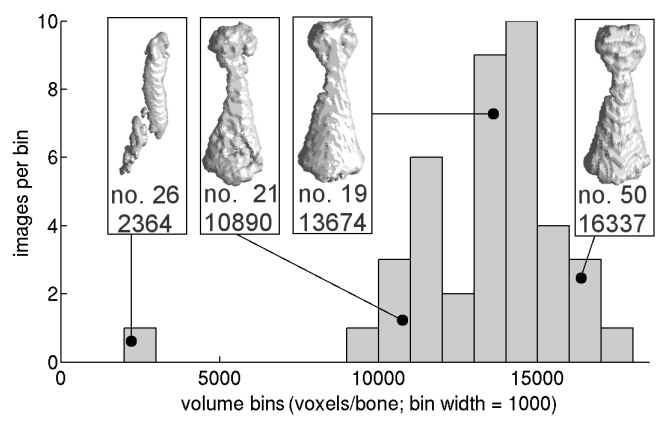

Fig. 12. Histogram of the segmented volumes of the middle finger medial phalanx in different MRI images. Surface renderings of four examples are shown, with the image numbers and volumes in number of voxels.

work are selected so that for each joint, the extremes and some intermediate positions are covered. The selection of postures influences the resulting model in the way that multiple recordings of similar joint postures assign them a greater weight compared to postures that occur only once.

In MRI acquisition, same tissue can be represented by different grey values. Artefacts such as missing parts, motion artefacts, artefacts due to the surrounding tissue and possibly distortions can occur. A discretisation error occurs due to the spacial resolution of $(0.76 \mathrm{~mm})^{3}$.

In the segmentation process, the segmented shape depends on the way the operator defines the grey value thresholds and manually refines the selection. The combined error of MRI acquisition and segmentation is illustrated by the distributions of grey value and segmented volume (Figures 11 and 12). An attempt was made at measuring the error of the MRI image acquisition by taking images of an animal bone without surrounding tissue, in order to discard the need for segmentation. However, the images showed hardly any signal, which might be due to the missing surrounding tissue or to a lack of humidity within the bone.
The motion estimation error depends on the quality of the segmented point clouds and the robustness of the algorithm with respect to differences in shape and grey value distribution. The combined error of steps 2 to 4 is partly expressed by the repeatability values in Table I, which however do not reflect a potential bias.

In this work, joints are modelled as rotational joints with constant parameters. In the case of a 1-DoF joint, this corresponds to rigid joint surfaces with perfectly circular cross-sections orthogonal to the joint axis. The 3-DoF joint with intersecting axes would be ideally represented by spherical joint surfaces. These are simplifications of the human joints with elastic cartilage and more complex surfaces.

The parameters of the defined joints are identified by way of numerical optimisations. These may introduce error by finding local optima. This error is assessed by the robustness of the result in the face of varying starting points (Section III-F).

\section{B. Joint types}

The model in the middle of Figure 7 seems to be the most natural one with two DoF for the thumb CMC and the MCP joints and one DoF for all other joints. The simpler model lacks a second DoF in the thumb $\mathrm{CMC}$ joint and in the middle finger MCP joint. On the other hand, the more complex model at the bottom has many additional DoF: in the thumb CMC and IP joint, in the PIP joints of index and little finger and in all the DIP joints. The tendon structure of the hand makes it seem unlikely or impossible that these axes represent independently actuated DoF. They probably compensate bone pose measurement errors or deviations of the bones from a perfectly circular path that really occur in the hand.

\section{Thumb CMC joint}

At the thumb CMC joint, the largest translational error occurs. This might be due to the fact that the thumb metacarpal poses are determined with respect to the index finger metacarpal. However, the bone that the thumb metacarpal articulates with is the trapezium bone, which is one of the carpal bones. Another possible explanation is that the motion of this joint is more complex, so that simple rotation axes are not sufficient to fully model it.

\section{Comparison between MRI and optical motion cap- ture}

Fitting a model with equal number of DoF to either MRI or optical motion capture (MoCap) data yielded no 
statistically significant differences in the mean residuals in three of four comparisons, and one statistically significant difference in favour of MoCap. This contradicts our initial hypothesis that MRI data can be fitted with significantly smaller residuals. The effect of the soft tissue artefact on MoCap data seems not to be as strong as initially postulated.

\section{ACKNOWLEDGEMENT}

The authors would like to thank Karolina Stonawska for the tedious work of segmenting the bones. This project was partly funded by the EU project The Hand Embodied (FP7-ICT-248587).

\section{REFERENCES}

[1] Wikipedia, "Hand," http://en.wikipedia.org/wiki/Hand, 2009.

[2] M. Grebenstein, A. Albu-Schäffer, T. Bahls, M. Chalon, O. Eiberger, W. Friedl, R. Gruber, U. Hagn, R. Haslinger, H. Höppner, S. Jörg, M. Nickl, A. Nothhelfer, F. Petit, J. Reill, N. Seitz, T. Wimböck, S. Wolf, T. Wüsthoff, and G. Hirzinger, "The DLR hand arm system," 2011 IEEE International Conference on Robotics and Automation, 2011.

[3] M. Grebenstein, M. Chalon, G. Hirzinger, and R. Siegwart, "A method for hand kinematics designers - 7 billion perfect hands," in Proceedings of 1st International Conference on Applied Bionics and Biomechanics, 2010.

[4] Y. Youm, T. E. Gillespie, A. E. Flatt, and B. L. Sprague, "Kinematic investigation of normal MCP joint," Journal of Biomechanics, vol. 11, pp. 109-118, 1978.

[5] K. N. An, E. Y. Chao, I. W. P. Cooney, and R. L. Linscheid, "Normative model of human hand for biomechanical analysis," Journal of Biomechanics, vol. 12, pp. 775-788, 1979.

[6] B. Buchholz, T. J. Armstrong, and S. A. Goldstein, "Anthropometric data for describing the kinematics of the human hands," Ergonomics, vol. 35, no. 3, pp. 261-273, 1992.

[7] A. Hollister, W. L. Buford, L. M. Myers, D. J. Giurintano, and A. Novick, "The axes of rotation of the thumb carpometacarpal joint," Journal of Orthopaedic Research, vol. 10, pp. 454-460, 1992.

[8] A. Hollister, D. J. Giurintano, W. L. Buford, L. M. Myers, and A. Novick, "The axes of rotation of the thumb interphalangeal and metacarpophalangeal joints," Clinical Orthopaedics and Related Research, vol. 320, pp. 188-193, 1995.

[9] J. L. Sancho-Bru, A. Pérez-González, M. Vergara-Monedero, and D. Giurintano, "A 3-D dynamic model of human finger for studying free movements," Journal of Biomechanics, vol. 34, pp. 1491-1500, 2001.

[10] G. S. Rash, P. Belliappa, M. P. Wachowiak, N. N. Somia, and A. Gupta, "A demonstration of the validity of a 3-D video motion analysis method for measuring finger flexion and extension," Journal of Biomechanics, vol. 32(12), pp. 13371341, 1999.

[11] L.-C. Kuo, F.-C. Su, H.-Y. Chiu, and C.-Y. Yu, "Feasibility of using a video-based motion analysis system for measuring thumb kinematics," Journal of Biomechanics, vol. 35, pp. 14991506, 2002.

[12] X. Zhang, L. Sang-Wook, and P. Braido, "Determining finger segmental centers of rotation in flexion-extension based on surface marker measurement," Journal of Biomechanics, vol. 36, pp. 1097-1102, 2003.
[13] P. Cerveri, N. Lopomo, A. Pedotti, and G. Ferrigno, "Derivation of centers of rotation for wrist and fingers in a hand kinematic model: Methods and reliability results," Annals of Biomedical Engineering, vol. 33, pp. 402-412, 2005.

[14] L. Y. Chang and N. S. Pollard, "Constrained least-squares optimization for robust estimation of center of rotation," Journal of Biomechanics, vol. 40, no. 6, pp. 1392 - 1400, 2007.

[15] _ , "Robust estimation of dominant axis of rotation," Journal of Biomechanics, vol. 40, no. 12, pp. 2707 - 2715, 2007.

[16] _ " "Method for determining kinematic parameters of the in vivo thumb carpometaracpal joint," IEEE Transactions on Biomedical Engineering, vol. 55(7), p. 1879ff, 2008.

[17] Dexmart, "Deliverable D1.1 kinematic model of the human hand," Dexmart, Tech. Rep., 2009.

[18] J. H. Ryu, N. Miyata, M. Kouchi, M. Mochimaru, and K. H. Lee, "Analysis of skin movement with respect to flexional bone motion using mr images of a hand," Journal of Biomechanics, vol. 39, pp. 844-852, 2006.

[19] K. Oberhofer, K. Mithraratne, N. Stott, and I. Anderson, "Error propagation from kinematic data to modeled muscle-tendon lengths during walking," Journal of Biomechanics, vol. 42, pp. 77-81, 2009.

[20] N. Miyata, M. Kouchi, M. Mochimaru, and T. Kurihaya, "Finger joint kinematics from MR images," in IEEE/RSJ International Conference on Intelligent Robots and Systems, 2005.

[21] M. Grebenstein and P. van der Smagt, "Antagonism for a highly anthropomorphic hand-arm system," Advanced Robotics, vol. 22(1), pp. 39-55, 2008.

[22] S. L. Delp, F. Anderson, S. Arnold, P. J. Loan, A. Habib, C. John, E. Guendelman, and D. Thelen, "OpenSim: Opensource software to create and analyze dynamic simulations of movement," IEEE Transactions on Biomedical Engineering, vol. 54, pp. 1940-1950, 2007.

[23] R. V. Gonzales, T. S. Buchanan, and S. L. Delp, "How muscle architecture and moment arms affect wrist flexion-extension moments," Journal of Biomechanics, vol. 30, pp. 705-712, 1997.

[24] A. Kapandji, "Cotation clinique de l'opposition et de la contreopposition du pouce [clinical test of opposition and counteropposition of the thumb]," Annales de Chirurgie de la Main, vol. 5(1), pp. 67-73, 1986.

[25] U. Hillenbrand, "Non-parametric 3D shape warping," in Proceedings International Conference on Pattern Recognition (ICPR), 2010.

[26] — , "Consistent parameter clustering: definition and analysis," Pattern Recognition Letters, vol. 28, pp. 1112-1122, 2007.

[27] U. Hillenbrand and A. Fuchs, "An experimental study of four variants of pose clustering from dense range data," pp. 1427 1448, 2011. [Online]. Available: http://www.sciencedirect.com/ science/article/pii/S1077314211001445

[28] B. K. Horn, "Closed-form solution of absolute orientation using unit quaternions," Journal of the Optical Society of America A, vol. 4(4), pp. 629-642, 1987.

[29] K. Fukunaga and L. D. Hostetler, "The estimation of a gradient of a density function, with applications in pattern recognition," IEEE Transactions on Information Theory, vol. 21, pp. 32-40, 1975.

[30] D. Comaniciu and P. Meer, "Mean shift: a robust approach toward feature space analysis," IEEE Transactions Pattern Analysis and Machine Intelligence, vol. 24, pp. 603-619, 2002.

[31] Wikipedia, "Hinge joint," http://en.wikipedia.org/wiki/Hinge_joint, 2006.

[32] J. A. Nelder and R. Mead, "A simplex method for function minimization," The Computer Journal, vol. 7, no. 4, pp. 308313, 1965. 
[33] A. Kapandji, The Physiology of the Joints. Edinburgh: Churchill Livingstone, 1998. 